\title{
A novel partner of Scalloped regulates Hippo signaling via antagonizing Scalloped-Yorkie activity
}

Tong Guo ${ }^{1, *}$, Yi Lu ${ }^{1, *}$, Peixue $\mathrm{Li}^{1}$, Meng-Xin Yin ${ }^{1}$, Dekang Lv ${ }^{1}$, Wenjing Zhang ${ }^{1}$, Huizhen Wang ${ }^{1}$, Zhaocai Zhou ${ }^{1}$, Hongbin $\mathrm{Ji}^{1}$, Yun Zhao ${ }^{1}$, Lei Zhang ${ }^{1}$

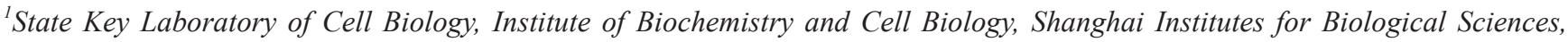
Chinese Academy of Sciences, 320 Yue-Yang Road, Shanghai 200031, China

The Hippo (Hpo) pathway controls tissue growth and organ size by regulating the activity of transcriptional co-activator Yorkie (Yki), which associates with transcription factor Scalloped (Sd) in the nucleus to promote downstream target gene expression. Here we identify a novel protein Sd-Binding-Protein (SdBP)/Tgi, which directly competes with Yki for binding to Sd through its TDU domains and inhibits the Sd-Yki transcriptional activity. We also find that SdBP retains Yki in the nucleus through the association with Yki WW domains via its PPXY motifs. Collectively, we identify SdBP as a novel component of the Hpo pathway, negatively regulating the transcriptional activity of SdYki to restrict tissue growth.

Keywords: Hippo; $\mathrm{Sd}$

Cell Research (2013) 23:1201-1214. doi:10.1038/cr.2013.120; published online 3 September 2013

\section{Introduction}

Among developmental processes, organ size control is one of the critical steps. Failure of appropriate organ size control results in diseases including tumorigenesis. In the past decade, the Hippo (Hpo) signaling pathway was identified as one of the important signaling pathways that regulate organ growth [1-4]. This pathway was first identified in Drosophila and is highly conserved in mammals. The serine/threonine Ste20-like kinase Hpo and the nuclear Dbf-2-related (NDR) family kinase Warts (Wts) associate with two scaffold proteins Salvador (Sav) and Mob as tumor suppressor (Mats) to induce a series of phosphorylation events, which is considered to be the core of the Hpo pathway [5-12]. This kinase cascade results in the inactivation and cytoplasmic retention of transcriptional co-activator Yorkie (Yki, YAP in mammals) $[13,14]$. In the absence of Hpo signaling, Yki translocates into the nucleus and binds to the transcrip-

\footnotetext{
*These two authors contributed equally to this work.

Correspondence: Lei Zhang ${ }^{\mathrm{a}}$, Yun Zhao ${ }^{\mathrm{b}}$

aE-mail: rayzhang@sibcb.ac.cn

bE-mail: yunzhao@sibcb.ac.cn

Received 17 June 2013; revised 29 July 2013; accepted 29 July 2013; published online 3 September 2013
}

tion factors such as Scalloped (Sd, TEAD/TEF family of proteins in mammals) [15-17] to promote proliferation and inhibit apoptosis by regulating the expression of Hpo pathway target genes including diap1, cyclin E and bantam $[18,19]$.

$\mathrm{Sd}$ is the only TEAD/TEF protein family member in Drosophila [20]. Unlike Drosophila, mammals contain four closely related TEAD/TEF family members, TEAD1-TEAD4. They are expressed widely during development and regulate the development of various tissues, including heart, skeletal muscles, neural crest, notochord and trophoectoderm [21-25]. The function of Sd-Yki complex has been intensively studied. In mammals, the structure and function of TEAD-YAP were well characterized [26-28]. Recent studies revealed that mammalian TEAD proteins are involved in the regulation of cell proliferation by cooperating with YAP under the control of Hpo signaling [29-32]. Interestingly, both genetic studies in Drosophila and pharmacological studies in transgenic mice revealed that loss of $s d$ or inhibition of TEAD has minimal impact on normal tissue homeostasis and physiology, suggesting that $\mathrm{Sd} / \mathrm{TEAD}$ is required for Yki/YAP-mediated tissue overgrowth but is largely dispensable for normal tissue homeostasis [15, 16, 33].

As YAP has been implicated in organ overgrowth and various human cancers $[4,29,34]$, this interesting prop- 
erty of TEAD may provide us a good opportunity to develop pharmacologically viable strategy against the YAP oncoprotein. However, so far, hardly any component(s) that directly inhibit Sd-Yki/TEAD-YAP complex activity has been identified. In this study, we report the discovery of a novel Sd binding protein, Sd-Binding-Protein $(\mathrm{SdBP})$. We find that it directly interacts with $\mathrm{Sd}$ via its TDU domains and with Yki through its PPXY motifs. We also reveal that it serves as an inhibitor of Sd-Yki transcriptional complex. Moreover, we provide evidence that SdBP and Yki compete with each other for Sd binding to regulate the transcriptional activity of Sd-Yki complex, thereby keeping the homeostasis of tissue growth.

\section{Results}

\section{Identification of SdBP as an Sd-binding partner}

To gain understanding of the regulatory mechanism of Sd-Yki complex, we performed yeast two-hybrid (Y2H) analysis using Sd C-terminal fragment (208-440 aa) as a bait to screen the Drosophila embryo cDNA library. We found that a protein (named as Sd-Binding-Protein, SdBP, encoded by CG10741) associated with Sd in Y2H assay (Supplementary information, Figure S1A).

To verify the interaction between SdBP and $\mathrm{Sd}$ in vitro, we performed co-immunoprecipitation experiment using full-length Sd and SdBP in Schneider 2 (S2) cells. Strong binding between $\mathrm{Sd}$ and $\mathrm{SdBP}$ in vitro was observed (Figure 1A). Subsequent GST pull-down assay confirmed a direct interaction between Sd and SdBP (Supplementary information, Figure S1B). In addition, we found that SdBP is a nuclear protein and co-localizes with $\mathrm{Sd}$ in the nucleus when expressed by Gal4 driver MS1096 (Supplementary information, Figure S1CS1E"'). Taken together, these results identify SdBP as a novel binding partner of $\mathrm{Sd}$.

There are two annotated transcripts of SdBP in FlyBase (http://flybase.org/reports/FBgn0036373.html). Expression of either isoforms of SdBP, SdBP-RA and SdBP-RB, under the control of MS1096 resulted in growth inhibition (Supplementary information, Figure S1F-S1F" '). No distinguishable difference between SdBP-RA and SdBP-RB in function and subcellular localization was observed (Supplementary information, Figure S1F-S1G'); we chose SdBP-RB for further study.

\section{$S d B P$ overexpression antagonizes $S d$-Yki-induced tissue overgrowth}

To probe the function of SdBP, we first examined the expression pattern of SdBP in different tissues including eye discs and wing discs by immunostaining and in situ assay. An antibody of SdBP was generated and validated using immunostaining by expressing SdBP RNAi transgene under the control of hh-Gal4, which drives gene expression in the posterior compartment (P-compartment) of Drosophila imaginal discs (Supplementary information, Figure S2A-S2A"). We observed that SdBP is ubiquitously expressed in eye discs and wing discs without a specific pattern (Supplementary information, Figure S2B-S2C' and S2D-S2E'). It is known that Sd is a transcription factor, and forms a transcriptional complex with Yki to control tissue growth in the Hpo pathway [15-17]. As SdBP binds to and co-localizes with Sd in vitro and in vivo (Figure 1A and Supplementary information, Figure S1A-S1E"'), we performed analyses to investigate whether SdBP plays a role in regulating SdYki transcriptional activity. SdBP was subjected to a dual luciferase assay that can reflect Sd-Yki transcriptional activity. In S2 cells, coexpression of Yki and Sd activated the luciferase ( $L u c$ ) reporter gene, 3xSd2-Luc [15] (Figure 1B). Coexpressing SdBP sharply decreased the activity of Sd-Yki complex (Figure 1B), suggesting that SdBP influenced Sd-Yki activity. In addition, this inhibition is specific as only mild change was observed when we tested SdBP using Wnt and Hh reporters (Supplementary information, Figure S2F-S2G).

To probe the function of SdBP on Sd-Yki activity in vivo, we overexpressed SdBP under the control of GMRGal4 in Drosophila compound eyes. Overexpression of $U A S-S d B P$ posterior to morphogenetic furrow using the GMR-Gal4 driver resulted in mild growth defects in eyes, compared with controls (compare Figure 1D with 1C). Yki overexpression-induced overgrowth phenotype was completely suppressed by coexpression of SdBP (compare Figure 1F with 1E). Furthermore, the GMR$Y k i$-induced increase in BrdU (a DNA replication maker) incorporation was diminished by coexpression of SdBP (Figure 1C'-1F'). These results suggest that SdBP suppresses growth through antagonizing Sd-Yki activity.

Gain of function of SdBP inhibits tissue growth through regulating the Hpo pathway

To verify whether SdBP overexpression induced growth defects via regulating the Hpo pathway, we expressed SdBP in flip-out clones to examine the changes in the expression of the Hpo pathway target genes. We observed a downregulation of DIAP1 and Ex proteins in SdBP-overexpressed regions (Figure 2A-2B'). Similar changes in diap1-lacZ (an enhancer trap for diap1 that reflects its transcriptional level) and ex-lacZ signals (an enhancer trap for expanded in response to Hpo signaling [35]) were observed (Supplementary information, Figure S3A-S3B"). The microRNA bantam is a Hpo pathway target that promotes cell growth $[18,19]$. We found that 


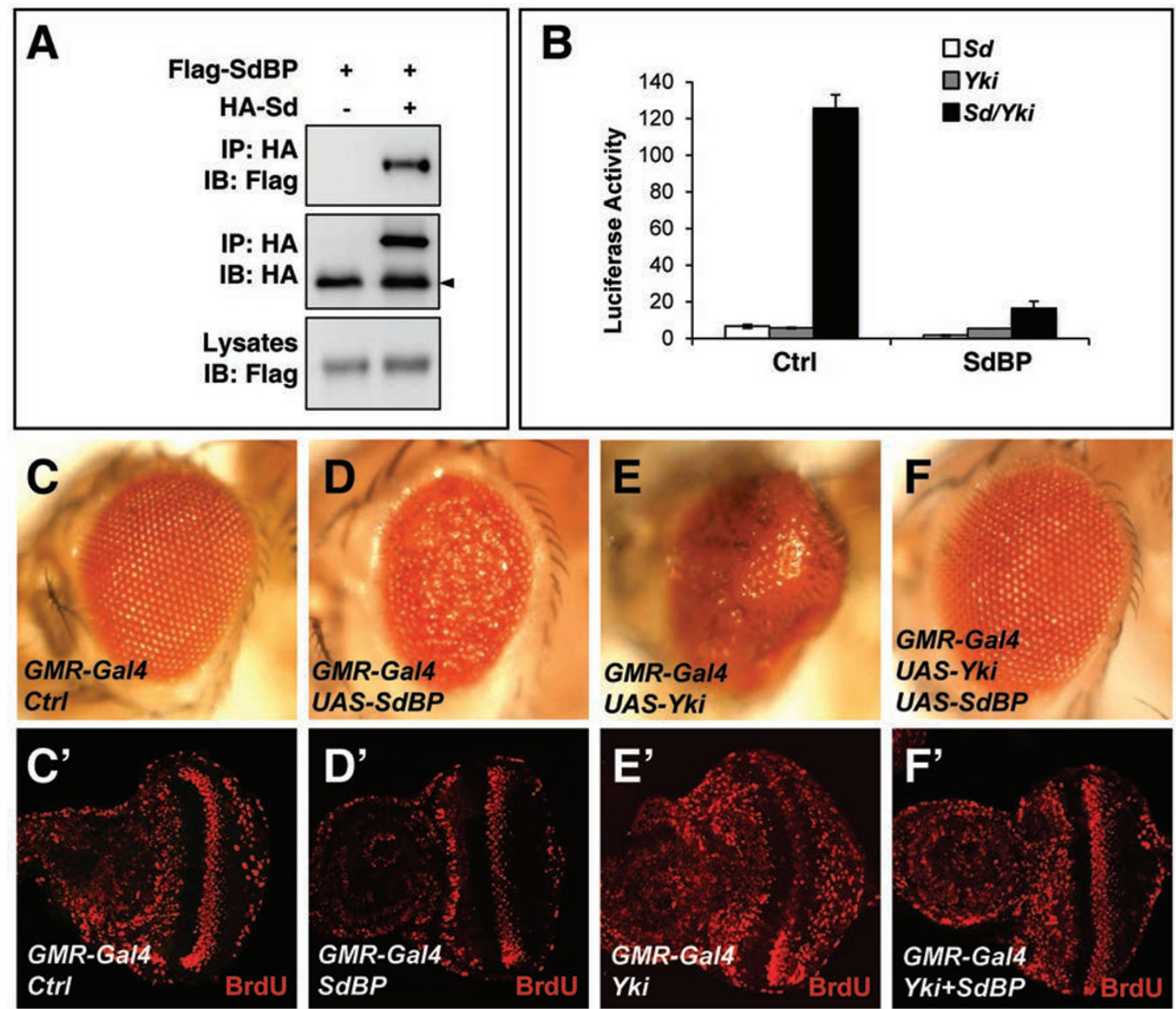

Figure $1 \mathrm{SdBP}$ associated with Sd and antagonized Sd-Yki-induced overgrowth. (A) Co-immunoprecipation of HA-Sd and 3x Flag-SdBP in S2 cells. The indicated constructs were transfected into S2 cells, followed by immunoprecipitation and western blot analysis with the indicated antibodies. The arrowhead indicates IgG chain. (B) Luciferase activity of a luciferase reporter plasmid with $3 \times$ Sd-binding sites and the control vector in S2 cells cotransfected with the indicated constructs. Data were presented as mean \pm s.d. $(n=3)$. $(C-F)$ Gain of function of SdBP induced growth defects and also reduced $G M R-Y k i$-induced overgrowth. Adult eyes expressing GMR-Gal4 (C), GMR-Gal4/UAS-SdBP (D), GMR-Gal4/UAS-Yki (E), and GMR-Gal4/UAS$(S d B P+Y k i)(F)$. (C'-F') BrdU staining of wild-type eye discs (C'), and eye discs expressing UAS-SdBP (D'), UAS-Yki (E') or UAS-(Yki+SdBP) (F') under the control of GMR-Gal4.

the expression of bantam-GFP reporter, which inversely correlates with the expression of bantam, was increased by overexpressing SdBP (Supplementary information, Figure S3C-S3C"). Briefly, SdBP inhibits tissue growth by restricting the expression of the Hpo pathway target genes.

To further dissect the function of SdBP in the Hpo pathway, we used mosaic analysis with a repressible cell marker (MARCM) system to express SdBP in hpo or sav mutant clones. We found that eye discs carrying $h p o^{B F 33}$ or $\operatorname{sav}^{S H 13}$ mutant clones exhibited overgrowth and elevated DIAP1 levels, resulting in enlarged and folded adult eyes (Figure 2C-2C"' and 2E-2E"'). Over- expressing SdBP in these mutant clones antagonized the overgrowth of mutant clones and the increase of DIAP1 in clone regions, resulting in nearly normal adult eyes (compare Figure 2D-2D" and 2F-2F" with 2C-2C" and $2 \mathrm{E}-2 \mathrm{E} "$ "). These pieces of evidence suggest that SdBP represses overgrowth phenotypes induced by loss of function of upstream Hpo signaling members.

TDU domains and PPXY motifs mediate the interactions of SdBP with Sd and Yki, respectively

We have demonstrated that SdBP induces growth suppression through antagonizing the activity of Sd-Yki complex. We next sought to investigate the mechanisms 

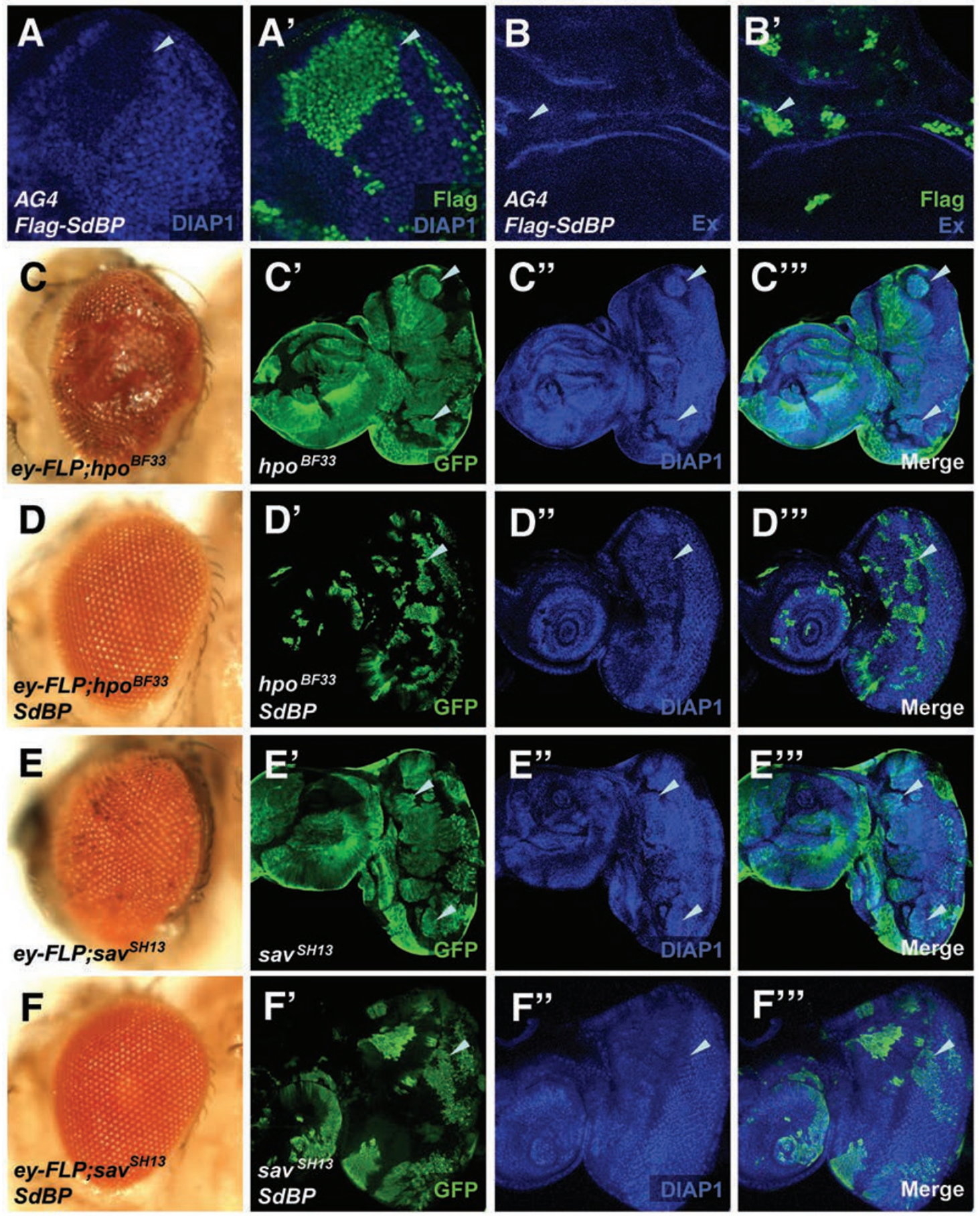

Figure 2 SdBP inhibited growth through downregulating the expression of the Hpo pathway target genes. (A-B') Overexpression of SdBP downregulated the expression of the Hpo pathway target genes under the control of act $>$ CD2 > Gal4 (AG4). SdBP overexpression region was marked by Flag (green). DIAP1 (A-A') and Ex (B-B') were stained (blue). Arrowheads indicated the clone regions. (C-F'") Eye discs bearing hpo mutants (C-C'”), hpo mutants overexpressing SdBP (D-D'”), sav mutants (E-E'"), and sav mutants overexpressing SdBP (F-F'"). GFP marked clones, and discs stained with DIAP1 (blue). Arrowheads indicated the clone regions.

by which SdBP regulates Sd-Yki activity. According to its primary sequence (Figure $3 \mathrm{~A}$ ), we generated several
SdBP variants that contained mutations in its two TDU domains. TDU domain is highly conserved and has been 

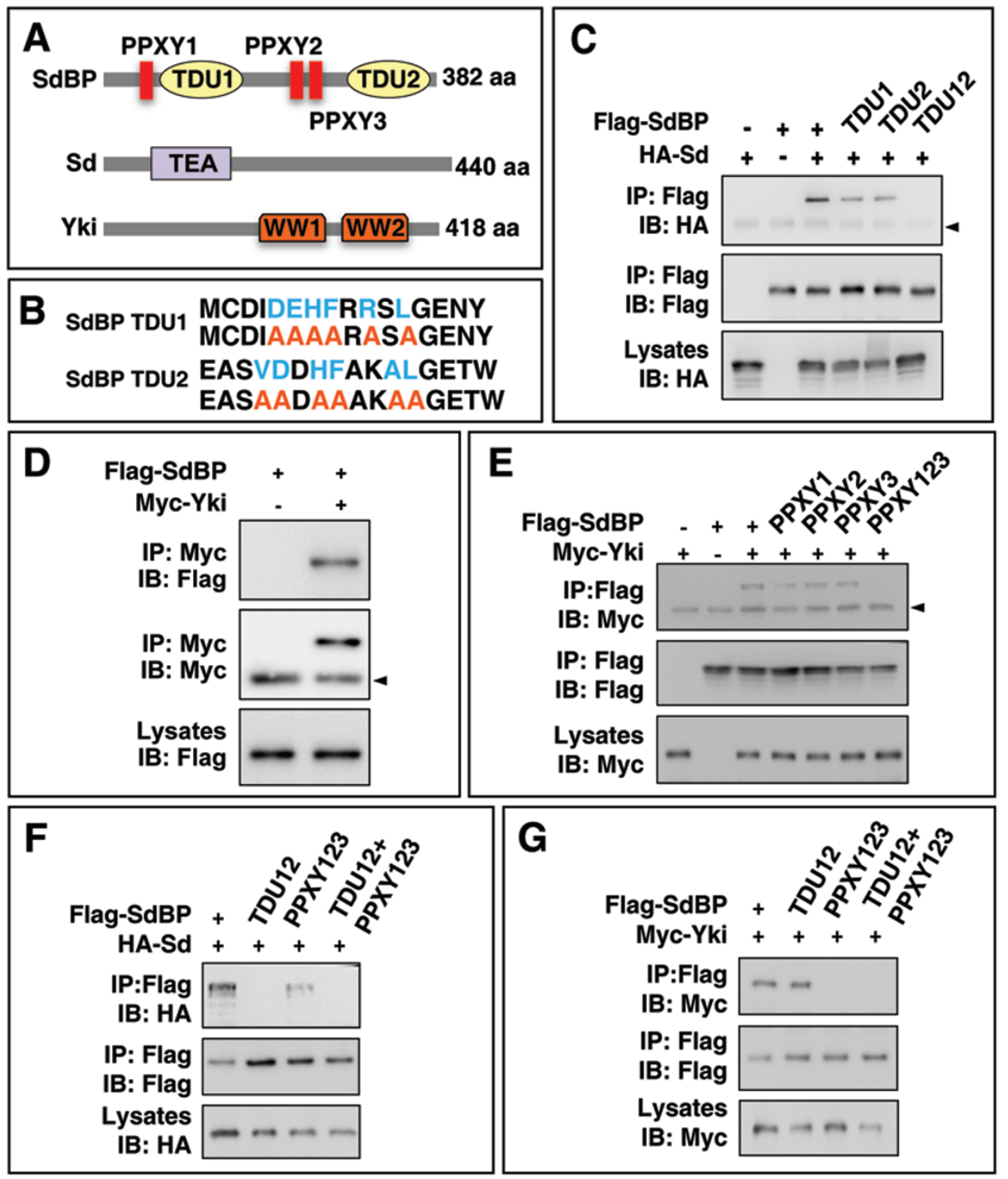

Figure 3 TDU domains and PPXY motifs mediated the interaction between SdBP and Sd/Yki. (A) Domain organization of SdBP, Sd and Yki. SdBP contains two TDU domains and three PPXY motifs, and Yki has two WW domains at its $C$ terminus. (B) TDU domain mutations generated in SdBP. Wild-type amino acids were shown in blue, and corresponding mutations were shown in red. (C) Co-immunoprecipation of Sd and the indicated SdBP TDU variants. The arrowhead indicates IgG chain. (D) Myc-Yki and 3× Flag-SdBP interacted with each other in S2 cells. The arrowhead indicates IgG chain. (E) Co-immunoprecipitation between Yki and the indicated SdBP PPXY variants. The arrowhead indicates IgG chain. (F, G) Co-immunoprecipitation assay showed that SdBP interacted with Sd and Yki variants. SdBP TDU variants only disrupted binding between SdBP and Sd, but not Yki (F), and vice versa (G). However, SdBP-(TDU12+PPXY123) bound with neither Sd nor Yki. The indicated constructs in C-G were co-transfected into S2 cells, followed by western blot assay directly or after immunoprecipitation.

shown to interact with TEAD/TEF family members [36]; therefore, we speculated that these two TDU domains might mediate the binding between SdBP and Sd. To verify this hypothesis, we performed co-immunoprecipitation and GST pull-down experiments using SdBP variants that carry mutations either in both TDU domains (TDU12) or in one of the TDU domains (TDU1 and TDU2) (Figure 3A-3C and Supplementary information, Figure S4A). The association between SdBP and Sd was completely abolished when both TDU domains were mutated, while 
the binding was still retained when single TDU domain was mutated (Figure 3C and Supplementary information, Figure S4A). These results suggest that both the TDU domains of SdBP are involved in the association of SdBP with Sd.

It was also reported that the Hpo pathway components utilize the WW domain and PPXY motif for proteinprotein interactions in a common way [37, 38]. Noticing that SdBP contains three PPXY motifs (Figure 3A), we speculated that a binding between SdBP and Yki through PPXY motifs and WW domains might exist. To verify this hypothesis, we performed co-immunoprecipitation and $\mathrm{Y} 2 \mathrm{H}$ assays. An interaction between SdBP and Yki was detected (Figure 3D and Supplementary information, Figure S4B). To confirm whether the binding is mediated by PPXY motifs and WW domains, SdBP variants carrying mutations either in all PPXY motifs (PPXY123) or in one of the PPXY motifs (PPXY1, PPXY2 and PPXY3) were used in co-immunoprecipitation (Figure 3E) and GST pull-down assays (Supplementary information, Figure S4C). We found that all three PPXY motifs are involved in mediating the binding between SdBP and Yki as only the triple mutation completely abolished the binding between SdBP and Yki (Figure 3E and Supplementary information, Figure S4C). In addition, deletion of both C-terminal WW domains of Yki abolished SdBPYki interaction (Supplementary information, Figure S4D). In brief, SdBP binds to Yki WW domains through its PPXY motifs.

To further explore the binding specificity between SdBP and Sd-Yki complex, we applied co-immunoprecipitation and GST pull-down assays, and found that TDU12 did not affect SdBP-Yki association, and PPXY123 did not affect SdBP-Sd interaction (Figure 3F and $3 \mathrm{G}$ and Supplementary information, Figure S4E), implying that SdBP-Sd association and SdBP-Yki association are independent of each other. Moreover, the SdBP variant carrying mutations in both TDU domains and all PPXY motifs (TDU12+PPXY123) did not bind to either Sd or Yki (Figure 3F and 3G and Supplementary information, Figure S4E). In addition, no direct interactions between SdBP and other known Hpo pathway components, such as Mer, Wts or Mats, were detected (Supplementary information, Figure S4F), suggesting that the function of SdBP in vivo in growth control is through its association with Sd-Yki complex.

TDU domains and PPXY motifs are critical for the function of SdBP in growth inhibition

We next focused on exploring whether the function of SdBP in growth inhibition was dependent on TDU domains and PPXY motifs. Using a dual luciferase as- say, we found that both SdBP variants, TDU12 and PPXY123, weakened the strength of the function of SdBP on Sd-Yki activity, whereas TDU12+PPXY123 no longer suppressed Sd-Yki activity (Figure 4A), implying that both TDU domains and PPXY motifs played important roles in regulating Sd-Yki activity in vitro. To further confirm these findings, we generated transgenic flies carrying different combination of mutations of these domains. Similar to what have been observed in luciferase assay (Figure 4A), when coexpressing SdBP variants with Yki under the control of GMR driver, only TDU12+PPXY123 was totally incapable of inhibiting the overgrowth phenotype induced by GMR-Yki (compare Figure 4G with 4B-4F). TDU12+PPXY123 might also have a dominant-negative function as its expression drove the eyes even larger and more folded than the GMR-Yki control (compare Figure 4G with 4C). When expressing the SdBP variants under the control of MS1096, TDU12 showed slightly reduced wings compared with control while PPXY123 led to smaller wings (Figure 4H-4K and 4M). Similar to what have been observed in eyes, TDU12+PPXY123 had a dominantnegative effect and resulted in even bigger wings (compare Figure 4L with $4 \mathrm{H}$, and $4 \mathrm{M}$ ). Meanwhile, we also tested the changes of downstream markers of the Hpo pathway when expressing these variants under the control of hhGal4 driver. Consistent with results presented above, TDU12 and PPXY123 decreased the protein level of DIAP1, while TDU12+PPXY123 led to no discernible change in DIAP1 level (compare Figure 4P-4P' and 4Q4Q' with 4N-4O' and 4R-4R'). Of note, expression of a TDU domain deletion form of SdBP (SdBP-TDUdel, in which both TDU domains were deleted) by $h h G a l 4$ also decreased the protein level of DIAP1 (Supplementary information, Figures S5), suggesting that the function of SdBP did not solely depend on TDU domains. Taken together, these findings indicate that both TDU domains and all PPXY motifs are involved in SdBP-induced growth inhibition.

\section{SdBP forms a complex with Sd and Yki in the nucleus}

We have demonstrated that SdBP binds to Sd and Yki independently and suppresses their activity, however, the detailed mechanism by which SdBP suppresses Sd-Yki activity is unclear. To figure out the underlying mechanism, we performed a two-step immunoprecipitation experiment. We found that $\mathrm{SdBP}, \mathrm{Sd}$ and Yki associate with each other and form a ternary complex (Figure $5 \mathrm{~A})$. To confirm this, we examined the localization of these proteins in Drosophila wing discs by overexpression. To clearly visualize the localization of these proteins, we generated GFP-fused SdBP (SdBP-GFP) for 

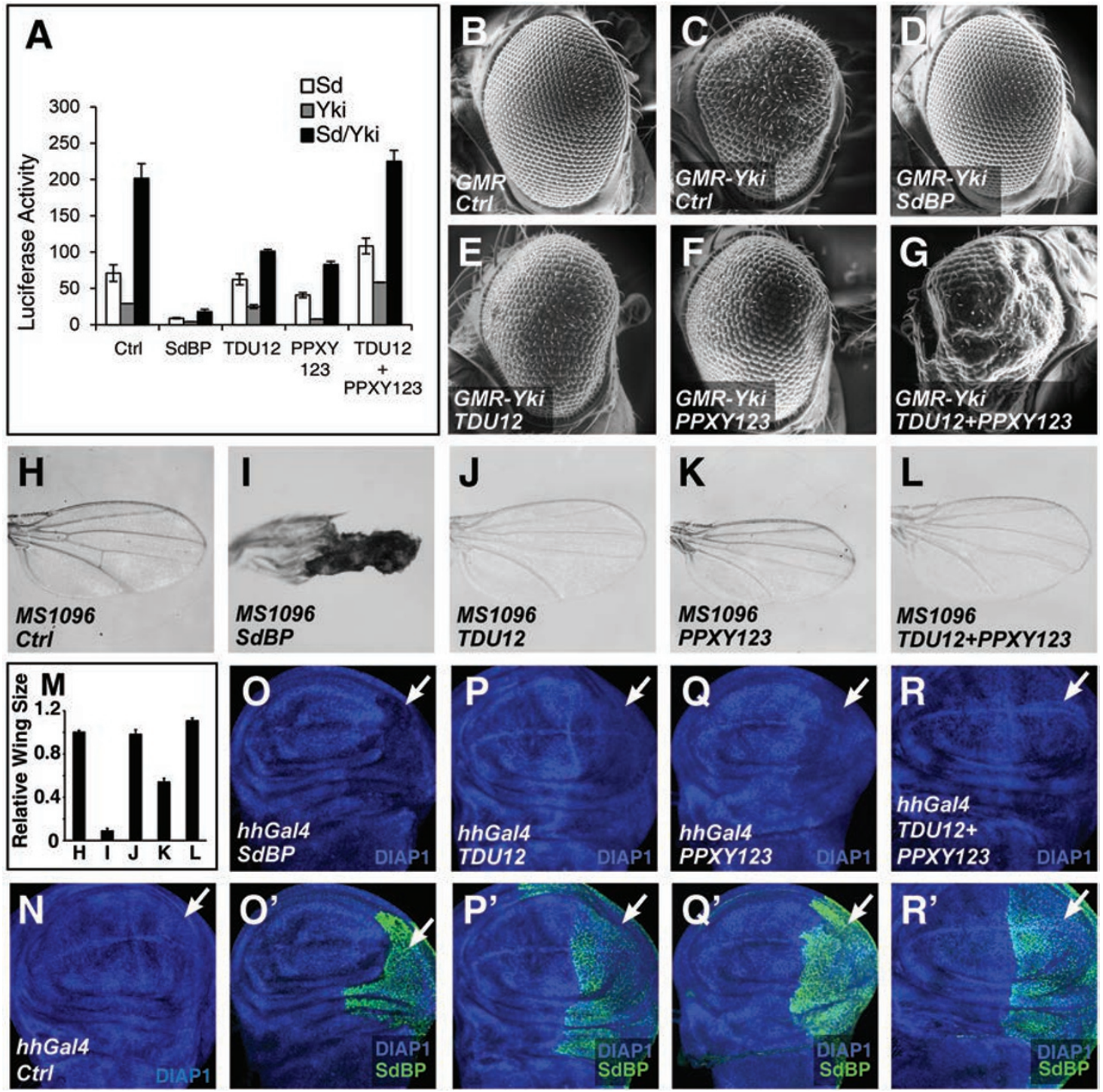

Figure 4 TDU domains and PPXY motifs are critical for SdBP function in growth inhibition. (A) Luciferase assay showed that SdBP variants inhibited Sd-Yki activity to different degrees. The indicated plasmids were co-transfected with $3 \times$ Sd luciferase reporter, and luciferase activity was measured by standard dual-luciferase protocols. Data were presented as mean \pm s.d. $(n=3)$. (B-G) Scanning electron microscope images of Drosophila compound eyes from the following genotypes: GMRGal4 (B), GMR-Gal4/UAS-Yki (C), GMR-Gal4/UAS-(Yki+SdBP) (D), GMR-Gal4/UAS-(Yki+TDU12) (E), GMR-Gal4/UAS(Yki+PPXY123) (F), and GMR-Gal4/UAS-(Yki+(TDU12+PPXY123)) (G). (H-L) Adult wings of wild-type (H), or wings expressing UAS-SdBP (I), UAS-TDU12 (J), UAS-PPXY123 (K), and UAS-(TDU12+PPXY123) (L) under the control of MS1096. (M) Quantification of relative wing sizes (fold change) of samples indicated in H-L. (N-R') Control wing discs (N) and wing discs expressing UAS-SdBP (O-O'), UAS-TDU12 (P-P'), UAS-PPXY123 (Q-Q') and UAS-(TDU12+PPXY123) (R-R') by hhGal4. SdBP (green) and DIAP1 (blue) were stained. Arrows indicated the P-compartment.

in vivo observation (Supplementary information, Figure S6A-S6A") and confirmed that there are no discernible functional differences between wild-type SdBP and SdBP-GFP (Supplementary information, Figure S6B-S6B"'). Interestingly, coexpression of SdBP-GFP and Yki resulted in an even distribution of Yki in cells unlike in the control where Yki mainly locates in the cytoplasm (compare Figure 5D and 5D" with 5B-5B'). This even distribution of Yki in cells was also observed when Sd and Yki were coexpressed (Figure 5C-5C'). When SdBP, Sd and Yki were coexpressed, the majority of Yki anchored in the nucleus (Figure 5E-5E" "), implying that Yki is retained in the nucleus upon SdBP coexpression. Moreover, expressing SdBP inhibited Sd- 

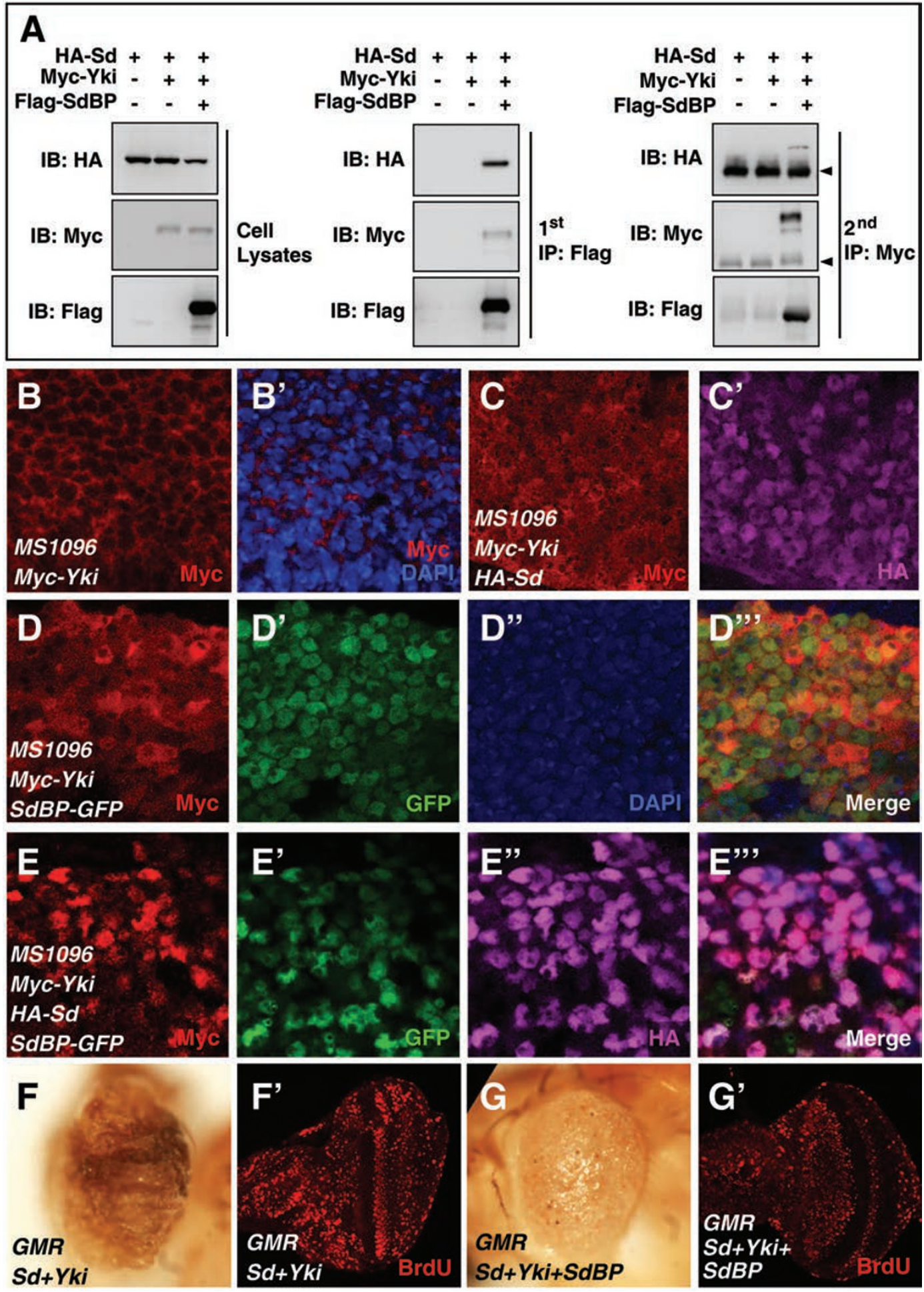

Figure 5 SdBP formed a complex with Sd-Yki. (A) S2 cells expressing the indicated proteins were collected for the two-step immunoprecipitation and analyzed by western blot. Cell lysates were first immunoprecipitated with M2-Flag beads, and then eluted for a second immunoprecipitation with Myc antibody. The arrowheads indicate IgG chain. (B-E'") Confocal images of third-instar wing discs expressing Myc-Yki (B-B'), or Myc-Yki with HA-Sd (C-C') or Myc-Yki with SdBP-GFP (D-D'”') or MycYki with HA-Sd and SdBP-GFP (E-E'"). Discs were stained with $\alpha$-Myc (red), $\alpha$-HA (magenta) antibodies and DAPI (blue). (FG') Adult eyes expressing GMR-Gal4/UAS-(Sd+Yki) (F) and GMR-Gal4/UAS-(Sd+Yki+SdBP) (G). BrdU staining of eye discs expressing UAS-(Sd+Yki) (F') and UAS-(Sd+Yki+SdBP) (G') under the control of GMR-Gal4. 
Yki overexpression-induced overgrowth of adult eyes as well as BrdU increase (Figure 5F-5G'), further indicating that SdBP suppresses the activity of Sd-Yki complex. In summary, SdBP retains Yki in the nucleus and simultaneously forms a complex with $\mathrm{Sd}$, while Sd-Yki activity is inhibited by the presence of SdBP.

\section{SdBP and Yki compete with each other for Sd to control tissue growth}

We showed that overexpressed SdBP forms a complex with Sd-Yki in the nucleus and inhibited their activity. To further explore the role of SdBP in the Hpo pathway, we generated a null mutation by "ends-out" gene targeting in which most of the $S d B P$ coding sequence was replaced by white gene [39]. Three independent null alleles, $s d b p^{A}, s d b p^{B}$ and $s d b p^{36}$, were isolated. Homozygotes of these alleles exhibit lethal phenotypes before the third instar larval stage. We used $s d b p^{B}$ allele to carry out further analyses (Supplementary information, Figure S7AS7A"). Interestingly, no discernible changes of clonal growth and the protein levels of DIAP1 and Ex were observed in the $s d b p^{B}$ mutant clones in eye and wing imaginal discs (Figure 6A-6A" and Supplementary information, Figure S7B-S7B"). Similar to the phenotypes induced by the loss of $s d$ in eye discs [15], loss of $s d b p$ did not affect the expression of the Hpo pathway downstream markers. In addition, loss of $s d b p$ did not affect the protein levels and subcellular localization of $\mathrm{Sd}$ or Yki (Supplementary information, Figure S7C-S7D"). It has been shown previously that $\mathrm{Sd}$ is absolutely required for tissue overgrowth induced by excessive Yki activity and loss of Sd suppresses the overgrowth phenotype as well as ectopic Hpo target gene expression caused by Yki overexpression [15]. Nevertheless, compared with Yki overexpression control (Figure 6B-6B" "), loss of $s d b p$ exhibited neither decrease nor increase in clone size or ectopic diap1 expression induced by Yki overexpression in $s d b p^{B}$ MARCM clones (Figure 6C-6C"'). Altogether, although neither $s d$ nor $s d b p$ is essential for normal eye development, the difference between them is that loss of $s d b p$ does not influence the phenotypes induced by excessive Yki activity in eye imaginal discs.

In order to explain how the transcriptional activity of nuclear Yki is inhibited by SdBP coexpression, we performed dosage-dependent GST pull-down and co-immunoprecipitation assays. We found that, upon SdBP binding, wild-type SdBP did not affect the stability of Sd-Yki complex while its variant carrying PPXA mutation in all PPXY motifs (SdBP-PPXY123) competed with Yki for Sd (Figure 6D and Supplementary information, Figure S7E). Conversely, we found that, Yki also competed with SdBP-PPXY123 (Figure 6E and Supplementary information, Figure S7F). These findings suggest that SdBP and Yki compete with each other for Sd binding in a concentration-dependent manner. However, only SdBP PPXY variant shows the dual competition (Figure 6D6E). Instead, wild-type SdBP forms a complex with SdYki (Figure 5A). To further investigate this intriguing finding, we employed the fluorescence resonance energy transfer (FRET) assay to detect the influence exerted by $\mathrm{SdBP}$ on the protein-protein interaction between $\mathrm{Sd}$ and Yki. In FRET assay, the transfer of energy between cyan fluorescent protein (CFP) and yellow fluorescent protein (YFP) was measured. Fusion proteins with CFP fused to the N-terminus of $\mathrm{Sd}\left(\mathrm{CFP}^{\mathrm{N}}-\mathrm{Sd}\right)$ and YFP fused to the $\mathrm{N}$ terminus of $\mathrm{Yki}\left(\mathrm{YFP}^{\mathrm{N}}-\mathrm{Yki}\right)$ were generated to examine whether the interaction between Sd and Yki could be affected by coexpressing SdBP in $\mathrm{S} 2$ cells. We observed that the FRET efficiency was decreased between $\mathrm{CFP}^{\mathrm{N}}$ Sd and $\mathrm{YFP}^{\mathrm{N}}-\mathrm{Yki}$ when SdBP was cotransfected (Figure $6 \mathrm{~F}$ ), suggesting that SdBP competes with Yki for Sd while simultaneously interacts with Yki via its PPXY motifs, resulting in the nuclear retention and dysfunction of Yki protein.

\section{Discussion}

The evolutionarily conserved Hpo signaling pathway has emerged as a pivotal pathway that plays a critical role in controlling tissue growth and organ size. The Sd-Yki

Figure 6 SdBP and Yki competed with each other for Sd to control growth. (A-C'”) Confocal images of third-instar wing discs bearing $h s-F L P$ clones of $s d b p^{B}$ mutant (A-A'"), GFP-labeled MARCM clones with Yki overexpression (B-B'"'), and GFPlabeled MARCM clones of $s d b p^{B}$ with Yki overexpression (C-C"'). Cells in clone regions were labeled by SdBP (red), Yki (red), DIAP1 (blue) and GFP (green). (D) GST pull-down assay showed that wild-type SdBP did not affect the stability of SdYki complex while SdBP-PPXY123 competed with Yki for Sd. His-YkiC plus dosage increased wild-type SdBP or SdBPPPXY123 was pulled down by GST-Sd, followed by western blot using the indicated antibodies. (E) GST pull-down assay showed that Yki also competed with SdBP-PPXY123 for Sd. Wild-type SdBP or SdBP-PPXY123 plus dosage increased HisYkiC were pulled down by GST-Sd, followed by western blot using the indicated antibodies. (F) S2 cells were cotransfected with CFP ${ }^{\mathrm{N}}$-Sd and YFP $^{\mathrm{N}}$-Yki. The FRET efficiency was decreased in the presence of SdBP. Data are presented as mean \pm S.d. $(n \geq 12)$. (G) A model of how Hpo signaling regulates the competition between Yki and SdBP for Sd binding. See discussion for details. 

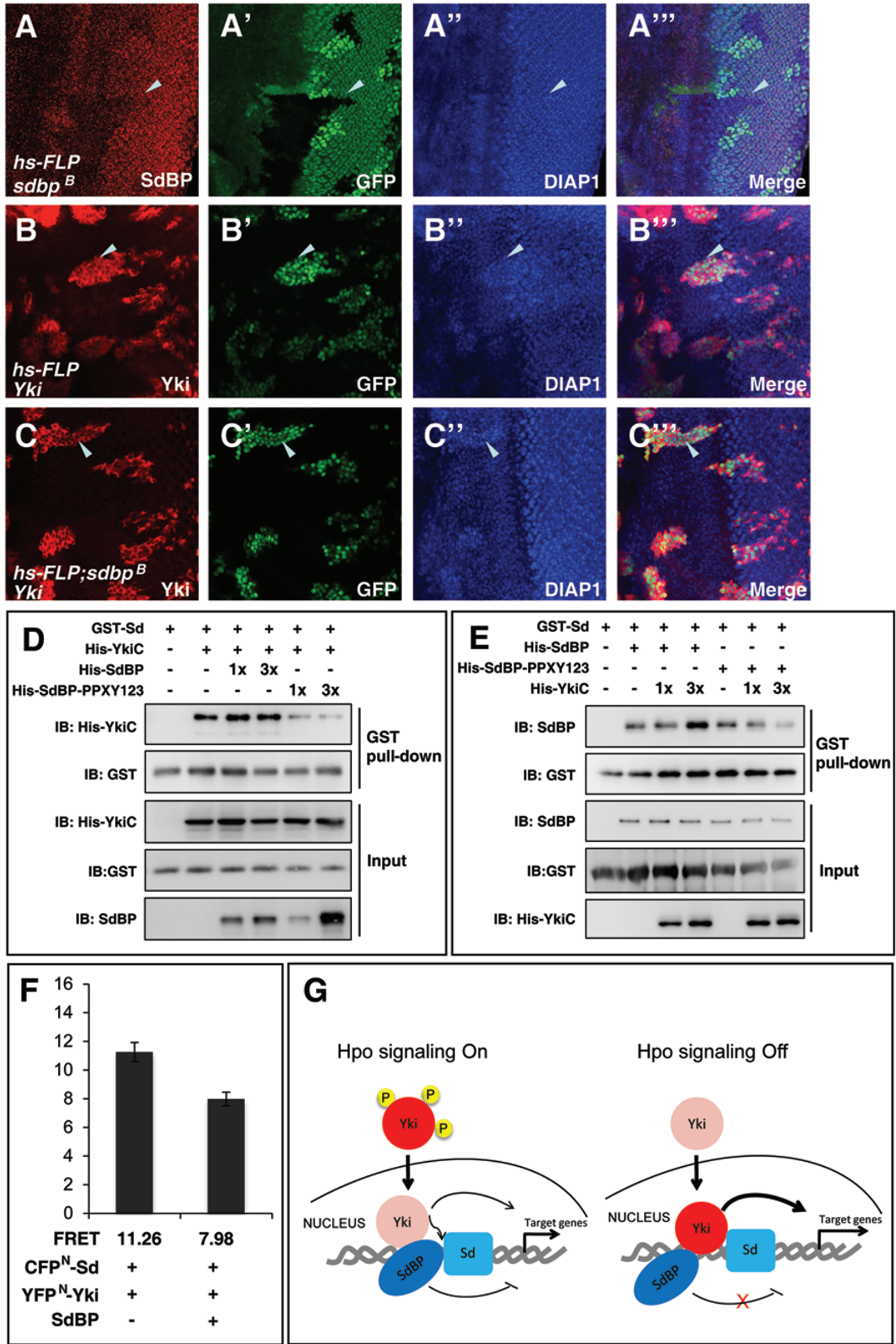

G

Hpo signaling On

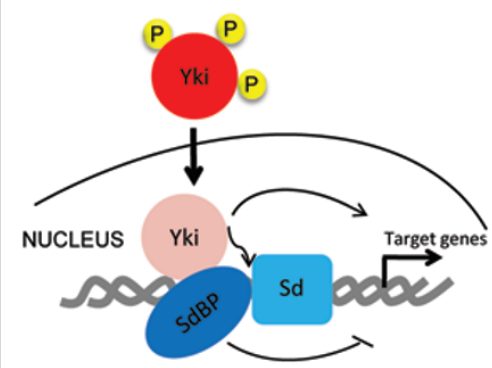

Hpo signaling Off

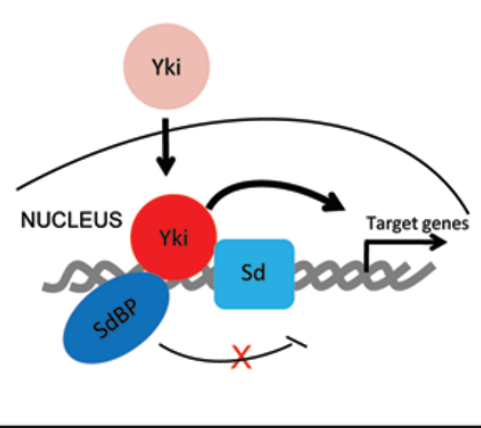


transcription complex serves as an essential downstream transcriptional effector and regulates Hpo target gene expression. In this study, using genetic and biochemical analyses, we identified SdBP as a novel negative regulator of Sd-Yki complex and found that it suppresses cell growth. Our data show that SdBP simultaneously binds to Sd through TDU domains and Yki via PPXY motifs (Figure 6). This association disrupts the direct interaction between Yki and Sd (Figure 6F), but results in a transcriptional inactivated ternary complex to precisely regulate the direction and intensity of Hpo signaling. We reason that SdBP competes with Yki for the binding to $\mathrm{Sd}$, as a result, within the ternary Sd-SdBP-Yki complex, Yki binds to PPXY motifs of SdBP, leading to an inhibition of Yki activity. Indeed, ectopic SdBP expression clearly suppresses the activity of Yki despite its nuclear location (Figure 5).

We further uncover a mutual competition of SdBP and Yki in binding to Sd. SdBP and Yki compete for Sd with a balance in normal physiological condition, resulting in neither growth defects nor growth advances. The Yin-Yang harmonization might promote the relative equilibrium of Hpo signaling in growth control. In the normal physiological condition, SdBP forms a repressor complex with Sd through unknown mechanisms in the nucleus, while the majority of Yki proteins are restricted in the cytoplasm by the upstream Hpo signaling (Figure 6G). Of note, Yki should be more competitive compared with SdBP, suggested by the structure information of the YAP-TEAD complex and the Vgll1-TEAD complex [40]. Similar to the YAP/Vgll1 and TEAD complexes, Yki and SdBP may form interfaces 1 and 2 in the TEADinteracting regions and may compete for binding to the same surface on TEAD, yet, Yki may contain an extra interface 3 compared with SdBP. Therefore, a low concentration of nuclear Yki protein may be sufficient to antagonize SdBP for normal tissue growth. However, a small amount of nuclear Yki is essential for tissue homoeostasis under normal conditions as suggested by previous finding that $y k i$ mutant clones grew poorly [41]. This might be due to the fact that in the absence of Yki, the fine balance between Yki and SdBP is disturbed, thus $\mathrm{SdBP}$ readily binds to $\mathrm{Sd}$ for efficient repression due to its nuclear localization. On the other hand, in the absence of SdBP, the existing small amount of nuclear Yki proteins can hardly induce the excessive expression of the Hpo pathway target genes, while the majority of Yki proteins are restricted in the cytoplasm by Hpo signaling; therefore, no obvious growth advances were observed in $s d b p$ mutant clones (Figure 6A-6A" and Supplementary information, Figure S7B). In the absence of Hpo signaling or in the case of Yki overexpression, abundant Yki proteins translocate into the nucleus and dominate for $\mathrm{Sd}$ regardless of whether SdBP existed (Figure 6G), resulting in elevated expression of Hpo pathway target genes (Figures 2C-2C"', 2E-2E" and 6B-6C"').

It has been shown previously that $\mathrm{Sd}$ acts in conjunction with Vestigial $(\mathrm{Vg})$ and other transcription factors to promote wing development by directly regulating the expression of wing patterning genes $[42,43]$. In addition to its expression in wing discs as $\mathrm{Vg}, \mathrm{Sd}$ is also expressed in other tissues and plays a broader role than $\mathrm{Vg}$ during development. In conjunction with Yki, Sd may be required for the basal expression of genes that can be suppressed by Hpo signaling in other tissues. Importantly, $\mathrm{Sd}$ is required for tissue overgrowth by regulating the expression of genes involved in cell proliferation, cell growth, and apoptosis when Yki is hyper-activated [1517].

In mammals, four genes encode TDU domain-containing proteins. Vestigial like 1, 2 and 3 have only one TDU domain; vestigial like 4 (Vgll4/Vg14) has two TDU domains. It has been reported that Vgll4 is the mammalian homolog of SdBP and binds to TEAD [44]. Interestingly, we identified that Vgll4 was frequently downregulated in human lung cancer specimens as a novel tumor suppressor through the direct competition with YAP for binding to TEAD4 (Zhang WJ et al., in submission). In contrast to SdBP, Vgll4 has 2 TDU domains but no PPXY motifs. Although Vgll4 cannot form a complex with YAP-TEADs in a way similar to SdBP, the competition of SdBP for Yki activity may be functionally and evolutionally conserved from Drosophila to mammals. This is consistent with the independent study by Koontz et al. showing that SdBP (named as Tondu-domain containing Growth Inhibitor (Tgi) in their study) and Vgll4 are conserved to work as negative regulators of Yki/YAP through a direct competition for Sd/TEAD2 binding [45].

Introduction of SdBP to Sd-Yki complex may bring us new ideas for the regulation of downstream Hpo pathway. As YAP is a known proto-oncogene in many cancers, the identification of SdBP/Vgll4 may provide us a promising therapeutic strategy for inhibiting YAP tumorigenic function in the future.

\section{Materials and Methods}

\section{Y2H screens}

Sd C-terminus (208-440 aa) fragment was used as baits to screen a Drosophila embryo cDNA library using the Matchmaker Gold Yeast Two-Hybrid system according to manufacturer's instructions (Clontech). SdBP-Sd interacting clones were selected for their ability to activate four independent reporter genes (AUR1$C, A D E 2, H I S 3$ and MEL1). The interaction between SdBP and Sd was further confirmed by co-immunoprecipitation. The interaction 
between SdBP and Yki was confirmed by $\mathrm{Y} 2 \mathrm{H}$ assays. pGBKT7$\mathrm{Sd}-\mathrm{C} / \mathrm{pACT} 2-\mathrm{Yki}$ and pGBKT7-Yki/pACT2-Sd were used as a positive control mating. pGBKT7-Sd-C/pACT2 and pGBKT7Yki/pACT2 were used as a negative control mating.

\section{Cloning of expression constructs}

SdBP cDNA or its variants were amplified by PCR and introduced into the pUAST-Flag or pUAST-3 $\times$ Flag vector by using the restriction enzymes $B g l \mathrm{II}$ and $X h o \mathrm{I}$. pUAST-Myc-Yki and pUASTHA-Sd constructs were previously described [15]. All PCR-amplified products were sequence-verified.

\section{Drosophila genetics}

All crosses and staging were done at $25^{\circ} \mathrm{C}$ unless specifically indicated. $U A S-S d B P$ or its variants flies were generated by cloning $S d B P$ or its variants after PCR amplification into the $p U A S T$, and injected into $y w$ flies. For flip-out ectopic expression clones, UAS-transgenes with Flag-SdBP were crossed to $A G 4$ flies. All lines gave qualitatively identical phenotypes. $S d B P R N A i$ stock (no. 34394) was provided by Bloomington Drosophila Stock Center (BDSC). HA-Sd;Myc-Yki recombinants were generated from the described stocks. Ectopic expression was induced in eyes using GMR-Gal4, in wings using MS1096, and in posterior cells using hhGal4. Mutant clones were generated using the MARCM system [46]. The genotypes for generated clones are as follows: (Figure 2C-2C"') ey-FLP/+; FRT42D hpo ${ }^{B F 33} / F R T 42 D$ tub-Gal80; +/tubGal4 UAS-GFP. (Figure 2D-2D"') ey-FLP/+; FRT42D hpo ${ }^{B F 33}$, FRT42D tub-Gal80; UAS-SdBP/tub-Gal4 UAS-GFP. (Figure 2E2E"') ey-FLP; UAS-GFP tub-Gal4/+; FRT82B sav ${ }^{\text {SH13 } / F R T 82 B ~}$ tub-Gal80. (Figure 2F-2F"') ey-FLP; UAS-GFP tub-Gal4/UASSdBP; FRT82B sav ${ }^{S H 13} / F R T 82 B$ tub-Gal80. (Figure 6B-6B"') hs-FLP tub-Gal4 UAS-GFP; UAS-Yki/+; FRT80B/FRT80B tubGal80. (Figure 6C-6C"') hs-FLP tub-Gal4 UAS-GFP; UASYki/+; FRT80B sdbp ${ }^{B} / F R T 80 B$ tub-Gal80. (Figure 6A-6A",, Supplementary information, Figure S7A", S7B-S7B", S7C-S7C", S7D-S7D") $h s-F L P ; ; F R T 80 B s d b p^{B} / F R T 80 B$ GFP. For generated SdBP mutant, SdBP knockout construct was generated according to the ends-out gene targeting strategy [39]. The targeting construct is expected to replace most of the coding sequence of $s d b p$ with white gene in pW25 vector. Two pairs of oligos: 1) 5'-ATTTGCGGCCGCCAGTTGCAGTTGCACTCAGGTTCGT-3' and 5'-ATTTGCGGCCGCGCTGTGGCTTTGCGGTTGATTTGGG-3' and 2) 5'-TTGGCGCGCCCCAATTCCCAACAGAACTCAACACA-3' and 5'-TTGGCGCGCCCAGCATTATTGTTGCTCGACGTGTT-3' were used to amplify DNA fragments of about $4.8 \mathrm{~kb}$ from Drosophila genomic DNA. These two fragments were cloned into the NotI and AscI sites of pW25, respectively. Flies carrying the targeting construct on the second chromosome were crossed to $y[1] w[*] ; P\{70 F L P\} 23$ P\{70I-SceI $\} 4$ A/TM6 (BDSC), and the following steps were performed as described previously [39].

Cell culture, transfection, co-immunoprecipitation, western blot, real-time PCR and luciferase reporter assays

Drosophila S2 cells were maintained in Schneider's medium supplemented with $10 \%$ fetal bovine serum at $25{ }^{\circ} \mathrm{C}$. Cells were transiently transfected using Lipofectamine (Invitrogen) according to the manufacturer's instructions. After $48 \mathrm{~h}$ of expression, cells were lysed in $1 \%$ Trition X-100 lysis buffer (50 mM Tris, $\mathrm{pH} 7.5$, $150 \mathrm{mM} \mathrm{NaCl}, 1 \%$ Triton X-100 and 1 mM EGTA) supplemented with Protease Inhibitor Cocktail (Roche). For co-immunoprecipitation, cells were lysed in NP-40 buffer (50 mM Tris-Cl, $\mathrm{pH}$ 8.0, 0.1 $\mathrm{M} \mathrm{NaCl}, 1 \%$ NP-40, 10\% Glycerol, $1.5 \mathrm{mM}$ EDTA, $10 \mathrm{mM}$ Sodium fluoride, $1 \mathrm{mM}$ Sodium vanadate, Protease Inhibitor Cocktail) for $30 \mathrm{~min}$ at $4{ }^{\circ} \mathrm{C}$. Lysates were incubated with the indicated antibodies for $2 \mathrm{~h}$ or overnight at $4{ }^{\circ} \mathrm{C}$. Samples were combined with $25 \mu$ l Protein A/G PLUS agarose (Santa cruz) for $1 \mathrm{~h}$ at $4{ }^{\circ} \mathrm{C}$. Beads were washed three times with NP-40 buffer, followed by western blot assay. For two-Step IP, Flag M2 affinity gel (Sigma) was used in the first-step IP, beads from the first IP were eluted with $3 \times$ Flag peptide (Sigma) and then mixed with Myc antibody in the second-step IP. Western blot was performed according to standard protocols. Protein gels were run and blotted using the Mini-PROTEAN Tetra Electrophoresis system (Biorad). Purified and associated proteins were detected by western blot analysis using chemiluminescence (Western Lightning Plus-ECL, PerkinElmer). Western blots were probed with antibodies against mouse $\alpha$-HA (Sigma), mouse $\alpha$-Flag (Sigma), mouse $\alpha$-Myc (Sigma), mouse $\alpha$-His (Sigma), and mouse $\alpha$-HRP (Santa cruz) using dilutions of 1:5 000 for all antibodies. Rabbit $\alpha$-SdBP antibody was generated using SdBP full-length protein, and used with dilution of 1:1 000 in western blot. Real-time PCR was performed using standard protocols. Luciferase reporter gene assay was performed using protocols as described previously [15].

\section{GST pull-down assay}

Glutathione agarose beads (GE) were used to purify the indicated proteins. These GST fusion beads were incubated with either S2 cell lysates or purified proteins in GST pull-down lysis buffer (20 mM Tris-Cl, pH 8.0, $200 \mathrm{mM} \mathrm{NaCl}, 1 \mathrm{mM}$ EDTA, 0.5\% NP-40 and $1 \%$ PMSF) at $4{ }^{\circ} \mathrm{C}$ for $1 \mathrm{~h}$, followed by washing three times with GST pull-down lysis buffer and western blot analysis.

\section{Immunostaining}

Wing and eye imaginal discs from third-instar larvae were dissected, fixed for $25 \mathrm{~min}$ in $4 \%$ formaldehyde and washed three times in PBS supplemented with $0.1 \%$ Triton X-100 (PBS-T). Discs were incubated in the primary antibody diluted in PBS-T for $2 \mathrm{~h}$ at room temperature, followed by three washes with PBS$\mathrm{T}$ and incubation with a secondary antibody in PBS-T for $2 \mathrm{~h}$ at room temperature. After three further washes, discs were mounted in PBS/glycerol medium with DAPI. Dilutions for the antibodies against mouse $\alpha$-Myc, mouse $\alpha$-HA, mouse $\alpha$-Flag, mouse $\alpha$-CD2 (Invitrogen) and rabbit $\alpha$-SdBP were 1:200. Antibodies: rabbit $\alpha$-Sd (1:50, produced by immunizing rabbits with the peptide of Sd amino acids 208-440) and rabbit $\alpha$-Yki (1:50, produced by immunizing rabbits with the peptide of Yki amino acids 180-418) were used. Fluorescent stains were captured on a Leica LAS SP5 confocal microscope.

\section{FRET assay}

$\mathrm{CFP}^{\mathrm{N}}$-Sd and $\mathrm{YFP}^{\mathrm{N}}$-Yki were transfected into S2 cells together with an ubi-Gal4 expression vector. Cells were washed with PBS, fixed with $4 \%$ formaldehyde for $20 \mathrm{~min}$, and mounted on slides in $80 \%$ glycerol. Fluorescence signals were acquired with the $63 \times$ objective of a Leica LAS SP5 confocal microscope. Each data set was based on 12-15 individual cells. In each cell, three to four regions of interest in photobleached area were selected for analysis. The intensity change of CFP was analyzed using the Leica soft- 
ware. The efficiency of FRET was calculated according to a previous paper [47].

\section{Acknowledgments}

We would like to thank Jinqiu Zhou and Margaret S Ho for their helpful discussion. We thank Jin Jiang, Wei Du, Bruce A Hay and Bloomington Stock Center for various reagents and stocks. This research was supported by the National Basic Research Program of China (973 Program; 2010CB912101, 2012CB945001, $2011 \mathrm{CB} 943902$ and 2011CB915502), the "Strategic Priority Research Program" of the Chinese Academy of Sciences (XDA01010406 and XDA01010405) and the National Natural Science Foundation of China (31171394 and 31171414). LZ is the scholar of the Hundred Talents Program of the Chinese Academy of Sciences.

\section{References}

1 Yin M, Zhang L. Hippo signaling: a hub of growth control, tumor suppression and pluripotency maintenance. J Genet Genomics 2011; 38:471-481.

2 Yu FX, Guan KL. The Hippo pathway: regulators and regulations. Genes Dev 2013; 27:355-371.

3 Irvine KD. Integration of intercellular signaling through the Hippo pathway. Semin Cell Dev Biol 2012; 23:812-817.

4 Harvey KF, Zhang X, Thomas DM. The Hippo pathway and human cancer. Nat Rev Cancer 2013; 13:246-257.

5 Harvey KF, Pfleger CM, Hariharan IK. The Drosophila Mst ortholog, hippo, restricts growth and cell proliferation and promotes apoptosis. Cell 2003; 114:457-467.

6 Pantalacci S, Tapon N, Leopold P. The Salvador partner Hippo promotes apoptosis and cell-cycle exit in Drosophila. Nat Cell Biol 2003; 5:921-927.

7 Udan RS, Kango-Singh M, Nolo R, Tao C, Halder G. Hippo promotes proliferation arrest and apoptosis in the Salvador/ Warts pathway. Nat Cell Biol 2003; 5:914-920.

8 Wu S, Huang J, Dong J, Pan D. hippo encodes a Ste-20 family protein kinase that restricts cell proliferation and promotes apoptosis in conjunction with salvador and warts. Cell 2003; 114:445-456.

9 Jia J, Zhang W, Wang B, Trinko R, Jiang J. The Drosophila Ste20 family kinase dMST functions as a tumor suppressor by restricting cell proliferation and promoting apoptosis. Genes Dev 2003; 17:2514-2519.

10 Lai ZC, Wei X, Shimizu T, et al. Control of cell proliferation and apoptosis by mob as tumor suppressor, mats. Cell 2005; 120:675-685.

11 Shimizu T, Ho LL, Lai ZC. The mob as tumor suppressor gene is essential for early development and regulates tissue growth in Drosophila. Genetics 2008; 178:957-965.

12 Wei X, Shimizu T, Lai ZC. Mob as tumor suppressor is activated by Hippo kinase for growth inhibition in Drosophila. EMBO J 2007; 26:1772-1781.

13 Zhao B, Wei X, Li W, et al. Inactivation of YAP oncoprotein by the Hippo pathway is involved in cell contact inhibition and tissue growth control. Genes Dev 2007; 21:2747-2761.

14 Ren F, Zhang L, Jiang J. Hippo signaling regulates Yorkie nuclear localization and activity through 14-3-3 dependent and independent mechanisms. Dev Biol 2010; 337:303-312.

15 Zhang L, Ren F, Zhang Q, et al. The TEAD/TEF family of transcription factor Scalloped mediates Hippo signaling in organ size control. Dev Cell 2008; 14:377-387.

16 Wu S, Liu Y, Zheng Y, Dong J, Pan D. The TEAD/TEF family protein Scalloped mediates transcriptional output of the Hippo growth-regulatory pathway. Dev Cell 2008; 14:388-398.

17 Goulev Y, Fauny JD, Gonzalez-Marti B, et al. SCALLOPED interacts with YORKIE, the nuclear effector of the hippo tumor-suppressor pathway in Drosophila. Curr Biol 2008; 18:435-441.

18 Nolo R, Morrison CM, Tao C, Zhang X, Halder G. The bantam microRNA is a target of the hippo tumor-suppressor pathway. Curr Biol 2006; 16:1895-1904.

19 Thompson BJ, Cohen SM. The Hippo pathway regulates the bantam microRNA to control cell proliferation and apoptosis in Drosophila. Cell 2006; 126:767-774.

20 Campbell S, Inamdar M, Rodrigues V, et al. The scalloped gene encodes a novel, evolutionarily conserved transcription factor required for sensory organ differentiation in Drosophila. Genes Dev 1992; 6:367-379.

21 Chen Z, Friedrich GA, Soriano P. Transcriptional enhancer factor 1 disruption by a retroviral gene trap leads to heart defects and embryonic lethality in mice. Genes Dev 1994; 8:2293-2301.

22 Milewski RC, Chi NC, Li J, et al. Identification of minimal enhancer elements sufficient for Pax3 expression in neural crest and implication of Tead 2 as a regulator of Pax3. Development 2004; 131:829-837.

23 Nishioka N, Inoue $\mathrm{K}$, Adachi $\mathrm{K}$, et al. The Hippo signaling pathway components Lats and Yap pattern Tead4 activity to distinguish mouse trophectoderm from inner cell mass. Dev Cell 2009; 16:398-410.

24 Sawada A, Kiyonari H, Ukita K, et al. Redundant roles of Tead 1 and Tead 2 in notochord development and the regulation of cell proliferation and survival. Mol Cell Biol 2008; 28:3177-3189.

25 Yagi R, Kohn MJ, Karavanova I, et al. Transcription factor TEAD4 specifies the trophectoderm lineage at the beginning of mammalian development. Development 2007; 134:38273836.

26 Li Z, Zhao B, Wang P, et al. Structural insights into the YAP and TEAD complex. Genes Dev 2010; 24:235-240.

27 Chen L, Chan SW, Zhang X, et al. Structural basis of YAP recognition by TEAD4 in the Hippo pathway. Genes Dev 2010; 24:290-300.

28 Tian W, Yu J, Tomchick DR, Pan D, Luo X. Structural and functional analysis of the YAP-binding domain of human TEAD2. Proc Natl Acad Sci USA 2010; 107:7293-7298.

29. Zhao B, Ye X, Yu J, et al. TEAD mediates YAP-dependent gene induction and growth control. Genes Dev 2008; 22:19621971.

30 Ota M, Sasaki H. Mammalian Tead proteins regulate cell proliferation and contact inhibition as transcriptional mediators of Hippo signaling. Development 2008; 135:4059-4069.

31 Cao X, Pfaff SL, Gage FH. YAP regulates neural progenitor cell number via the TEA domain transcription factor. Genes Dev 2008; 22:3320-3334.

32 von Gise A, Lin Z, Schlegelmilch K, et al. YAP1, the nuclear 
target of Hippo signaling, stimulates heart growth through cardiomyocyte proliferation but not hypertrophy. Proc Natl Acad Sci USA 2012; 109:2394-2399.

33 Liu-Chittenden Y, Huang B, Shim JS, et al. Genetic and pharmacological disruption of the TEAD-YAP complex suppresses the oncogenic activity of YAP. Genes Dev 2012; 26:13001305.

34 Pan D. The hippo signaling pathway in development and cancer. Dev Cell 2010; 19:491-505.

35 Hamaratoglu F, Willecke M, Kango-Singh M, et al. The tumour-suppressor genes NF2/Merlin and Expanded act through Hippo signalling to regulate cell proliferation and apoptosis. Nat Cell Biol 2006; 8:27-36.

36 Vaudin P, Delanoue R, Davidson I, Silber J, Zider A. TONDU (TDU), a novel human protein related to the product of vestigial (vg) gene of Drosophila melanogaster interacts with vertebrate TEF factors and substitutes for $\mathrm{Vg}$ function in wing formation. Development 1999; 126:4807-4816.

37 Ludes-Meyers JH, Kil H, Bednarek AK, et al. WWOX binds the specific proline-rich ligand PPXY: identification of candidate interacting proteins. Oncogene 2004; 23:5049-5055.

38 Webb C, Upadhyay A, Giuntini F, et al. Structural features and ligand binding properties of tandem WW domains from YAP and TAZ, nuclear effectors of the Hippo pathway. Biochemistry 2011; 50:3300-3309.

39 Gong WJ, Golic KG. Ends-out, or replacement, gene targeting in Drosophila. Proc Natl Acad Sci USA 2003; 100:2556-2561.

40 Pobbati AV, Chan SW, Lee I, Song H, Hong W. Structural and functional similarity between the Vgll1-TEAD and the YAPTEAD complexes. Structure 2012; 20:1135-1140.

41 Huang J, Wu S, Barrera J, Matthews K, Pan D. The Hippo signaling pathway coordinately regulates cell proliferation and apoptosis by inactivating Yorkie, the Drosophila Homo$\log$ of YAP. Cell 2005; 122:421-434.

42 Halder G, Polaczyk P, Kraus ME, et al. The Vestigial and Scalloped proteins act together to directly regulate wingspecific gene expression in Drosophila. Genes Dev 1998; 12:3900-3909.

43 Guss KA, Nelson CE, Hudson A, Kraus ME, Carroll SB. Control of a genetic regulatory network by a selector gene. Science 2001; 292:1164-1167.

44 Chen HH, Mullett SJ, Stewart AF. Vgl-4, a novel member of the vestigial-like family of transcription cofactors, regulates alpha1-adrenergic activation of gene expression in cardiac myocytes. J Biol Chem 2004; 279:30800-30806.

45 Koontz LM, Liu-Chittenden Y, Yin F, et al. The hippo effector yorkie controls normal tissue growth by antagonizing scalloped-mediated default repression. Dev Cell 2013; 25:388401.

46 Lee T, Luo L. Mosaic analysis with a repressible cell marker (MARCM) for Drosophila neural development. Trends Neurosci 2001; 24:251-254.

47 Centonze VE, Firulli BA, Firulli AB. Fluorescence Resonance Energy Transfer (FRET) as a method to calculate the dimerization strength of basic Helix-Loop-Helix (bHLH) proteins. Biol Proced Online 2004; 6:78-82.

(Supplementary information is linked to the online version of the paper on the Cell Research website.) 